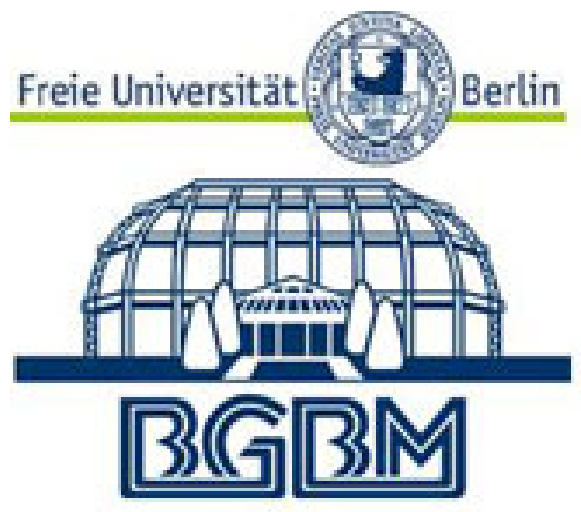

Neue Arten von Plantago, Sektion Cleiosantha und Novorbis Decne Author(s): R. Pilger

Source: Notizblatt des Königl. botanischen Gartens und Museums zu Berlin, Bd. 5, Nr. 49 (Jun. 27, 1912), pp. 259-263

Published by: Botanischer Garten und Botanisches Museum, Berlin-Dahlem

Stable URL: http://www.jstor.org/stable/3994437

Accessed: $14 / 06 / 2014$ 08:43

Your use of the JSTOR archive indicates your acceptance of the Terms \& Conditions of Use, available at http://www.jstor.org/page/info/about/policies/terms.jsp

JSTOR is a not-for-profit service that helps scholars, researchers, and students discover, use, and build upon a wide range of content in a trusted digital archive. We use information technology and tools to increase productivity and facilitate new forms of scholarship. For more information about JSTOR, please contact support@jstor.org. 


\section{Neue Arten von Plantago, Sektion Cleiosantha und Novorbis Decne.}

Von

R. Pilger.

Plantago accrescens Pilger n. sp.; elata, perennis, rhizomate valido, crasso, horizontali vel fere verticali; folia elongata basi vaginantia, angusta, lanceolata, superne sensim cuneatim angustata, inferne sensim in petiolum longiorem angustata, $25-40 \mathrm{~cm}$ longa, $25-30 \mathrm{~mm}$ lata, glabra, integra; folia in rosula inferiora minora, sensim accrescentia; pedunculi adscendentes validi ad $55-60 \mathrm{~cm}$ alti (cum spica ad $15 \mathrm{~cm}$ longa laxiuscula), inferne glabrescentes, superne villosi; bractea lanceolatoovata, parum margine ciliolata, $2,75-3 \mathrm{~mm}$ longa; sepala latiora rotundata vel rotundato-ovata, dorso ad nervum parum breviter pilosula, $3 \mathrm{~mm}$ longa, sepala angustiora obovato-ovalia, $2,5 \mathrm{~mm}$ longa; flores aperti, corollae laciniae ovatae, acutae, $2,5 \mathrm{~mm}$ longae; ovarium 3 -ovulatum.

Argentinien: Rioja, Sierra Famatina (Hieronymus et Niederlein n. 744. - Januar 1879).

Plantago alismatifolia Pilger $\mathrm{n}$. sp.; rhizoma verticale in radicem fusiformem transiens; folia anguste ovalia ad ovalia, superne breviter arcuatim angustata, inferne sensim in petiolum longum vel breviorem angustata, ad $17-20 \mathrm{~cm}$ (cum petiolo ad $7-8 \mathrm{~cm}$ longo) longa et ad $3-3,5 \mathrm{~cm}$ lata, margine subintegra vel remote distincte denticulata, laxe villis albidis longioribus inspersa; pedunculi ad $45 \mathrm{~cm}$ alti (cum spica ad $13 \mathrm{~cm}$ longa), patenter laxe villosi; spica laxiflora; flores clausi, staminodiis inclusis, stilo exserto, rarius flores aperti; bractea triangularis, villosa; sepala latiora late ovata, parum inaequilatera, superne breviter ciliolata, in nervo breviter pilosa, 3,5 mm longa, sepala angustiora ovalia, $3 \mathrm{~mm}$ longa; corollae laciniae ovatae, parum acutatae, $3,5 \mathrm{~mm}$ longae; semina in capsula 2, circ. $2 \mathrm{~mm}$ longa.

Mexico: (Schaffner n. 434); Santa Fé (Pringle n. 9297. September 1901).

Die Pringlesche Pflanze wurde als $P$. hirtella Kth. ausgegeben. Letztere Art, die mir nur in wenigen Exemplaren aus Ecuador und Kolumbien bekannt ist, ist bisher stets verkannt worden, auch in der Decaisneschen Bearbeitung. In einer vollständigen Bearbeitung der Sektion werde ich bald darüber näheres publizieren. 
Plantago subnuda Pilger n. sp.; rhizoma breve crassum; folia crassiuscula, subcoriacea, ovalia vel obovato-ovalia vel elliptica, superne breviter arcuato-cuneatim vel late cuneatim angustata, inferne sensim in petiolum longiorem vel breviorem angustata, $8-19 \mathrm{~cm}$ longa, 2,5 ad $4 \mathrm{~cm}$ lata, margine leviter remote denticulata, glabra vel villis nonnullis albidis vix inspersa; pedunculi validi ad $27 \mathrm{~cm}$ longi (cum spica $8 \mathrm{~cm}$ longa haud plane evoluta); spica densa; bractea ovato-ovalis, praeter marginem vix ciliolatum glabra, $3,5 \mathrm{~mm}$ longa; sepala latiora rotundatoovata, obtusa, inaequilatera, glabra, $3 \mathrm{~mm}$ parum superantia, sepala angustiora ovalia, $3 \mathrm{~mm}$ longa; ovarium 3 -ovulatum; flores clausi, stilus 土exsertus; corollae laciniae anguste ovatae, acutae, $3 \mathrm{~mm}$ longae.

Kalifornien: Monterey-County (Heller n. 6764. - März 1903).

Die Art wurde als $P$. hirtella Kth. ausgegeben (vergl. bei $P$. alismatifolia Pilger). Sie gehört in die Verwandtschaft von P. Candollei Rap.

Plantago hypolasia Pilger n. sp.; elata, annua, radice fusiformi, elongato; folia rosulata ovalia, superne breviter rotundato-cuneatim angustata, inferne sensim in petiolum angustata, ad $24 \mathrm{~cm}$ longa, ad $5 \mathrm{~cm}$ lata, margine subintegra vel remote parum denticulata; folia juniora dense lanato-villosa, folia adulta supra parum villosa, subtus et imprimis ad petiolum densius villosa; pedunculi erecti ad $50-60 \mathrm{~cm}$ longi (cum spica ad $35 \mathrm{~cm}$ longa), villosi; spica inferne laxa, superne densior; bractea anguste lanceolato-triangularis, pilis longis patentibus inspersa, $3 \mathrm{~mm}$ longa; sepala latiora late ovata ad rotundata, margine superne minutissime ciliolulata, ad nervum breviter piloso-setosula, parum inaequilatera, $3 \mathrm{~mm}$ longa, sepala angustiora anguste ovalia, obtusa, parum supra 2,5 mm longa; flos apertus vel clausus, corollae laciniae ovatae ad anguste ovatae, $3 \mathrm{~mm}$ longae; semina 3 evoluta.

Concepcion del Uruguay: (Dr. Lorentz Flora Entreriana n. 1124. - September 1877).

Die neue Art ist aus der Verwandtschaft von P. tomentosa Lam.

Plantago Kurtzii Pilger n. sp.; videtur perenne, rhizoma in spec. haud conservatum; folia crassiuscule coriacea, elliptica, breviuscule arcuato-cuneatim in petiolum brevem angustata, superne late cuneatim angustata, margine integra, glabra, $15-16 \mathrm{~cm}$ longa, 4,5-5 cm lata, venis reticulatis bene conspicuis instructa; pedunculus unicus tantum in specim., $37 \mathrm{~cm}$ altus (cum spica $12 \mathrm{~cm}$ longa), validus, erectus, inferne glabratus, superne albido-villosus; spica crassa, densa, basi tantum laxa; bractea ovato-ovalis, margine breviter villosa, $3 \mathrm{~mm}$ longa; sepala latiora rotundata, inaequilatera, $3,25-3,5 \mathrm{~mm}$ longa, margine et apice parum ciliolata, sepala angustiora ovali-elliptica $3 \mathrm{~mm}$ longa; flores aperti, corollae laciniae (ad capsulam fere maturam tantum visae) late 
ovatae, acutae, 2,5 mm longae; capsula 3-sperma vel ovalo haud evoluto saepe 2-sperma; semina ad 2,5 mm longa.

Argentinien: Mendoza, am Rio Salado (F. Kurtz n. 7087. Januar 1892).

Plantago nigritella Pilger n. sp.; parva, annua, sicca nigricans; radix fusiformis satis elongatus; folia lanceolata ad ovali-lanceolata, superne breviter arcuato-cuneatim angustata, inferne in petiolum longitudine satis variantem sensim angustata, $3-6 \mathrm{~cm}$ longa, ad $13 \mathrm{~mm}$ lata, subintegra vel 士 leviter denticulata, adulta pilis albidis satis rigidis villosa, juniora villoso-hirsuta; pedunculi breves, arcuatim adscendentes, ad $4 \mathrm{~cm}$ longi (cum spica $1,5-2 \mathrm{~cm}$ longa), hirsuto-villosi, rhachis longa villosa; bractea latius triangularis, dorso pilis longis inspersa, $2 \mathrm{~mm}$ longa; calyx glabratus praeter cilias parvas ad marginem superiorem, nonnumquam et pilis parvis in dorso inspersus; sepala latiora rotundato-ovata, e nervo vix acutata, $2 \mathrm{~mm}$ longa, sepala angustiora ovalia parum breviora; flores clausi, staminodia parum evoluta, corollae laciniae ovatae vel anguste ovatae, $2 \mathrm{~mm}$ vel parum supra longae; ovarium 3-ovulatum.

Argentinien: Cordoba, Rio primero (Th. Stuckert n. 11844. - Oktober 1902).

Die neue Art ist aus der Verwandtschaft von $P$. myosuros Lam.

Plantago Pflanzii Pilger n. sp.; rhizoma breve indivisum vel etiam breviter ramosum, rosulan sterilem lateralem procreans; folia subcoriacea, anguste ovalia vel ovalia, superne arcuato-cuneatim angustata, inferne sensim in petiolum latum breviorem late vaginantem angustata, ad $20 \mathrm{~cm}$ longa, $3-4 \mathrm{~cm}$ lata, margine subintegra vel parum obtusiuscule dentata, villis brevioribus \pm adpressis parum inspersa, margine saepius quasi albido-ciliata; pedunculi erecti ad $42 \mathrm{~cm}$ alti (cum spica ad $16 \mathrm{~cm}$ longa), laxe villis longioribus obsiti; spica densa basi tantum laxiuscula; bractea ovato-ovalis, $2,5 \mathrm{~mm}$ longa, margine ciliolata, dorso pilis rigidulis obsita; sepala latiora lata, fere rotundata, $3,25 \mathrm{~mm}$ longa, margine superne brevissime ciliolata, sepala angustiora ovalia, 2,75 mm longa; flores aperti, corollae laciniae late ovatae, $2 \mathrm{~mm}$ vel parum supra longae; capsula 3 -sperma, semina 2-2,25 mm longa.

Bolivien: Palca-La Paz, Huancapampa (Pflanz n. 442 A, 444 C. - Februar 1910); ibid. (Pflanz n. 321. - Fruchtend im Juli 1909).

Die Art zeigt das in der Gruppe seltene Verhalten, daB öfters am Rhizom Verzweigung eintritt, indem eine seitliche Rosette gebildet wird, die aber mit der Grundachse in dichtem Zusammenhang bleibt.

Plantago refracta Pilger n. sp., perennis, rhizomate brevi crasso; folia subcoriacea, ovalia vel oblanceolato-ovalia, superne breviter, 
anguste vel latius arcuatim angustata, apice ipso obtusa, callosoincrassata et breviter refracta, inferne sensim in petiolum longiorem angustata, integra, glabra, margine tantum hic illic pilis brevibus nonnullis obsita, 20-26 cm longa, $3-4 \mathrm{~cm}$ lata; pedunculi erecti ad $50 \mathrm{~cm}$ alti (cum spica ad $23 \mathrm{~cm}$ longa), inferne glabrati, superne villosi; spica densa, basi tantum laxiuscula; bractea ovali-ovata, 2,75-3 mm longa, margine parum ciliolulata; sepala latiora rotundata vel ovatorotundata, inaequilatera, $2,5 \mathrm{~mm}$ longa, margine vix lacerulato-ciliolulata, ad nervum scaberula, sepala angustiora obovato-ovalia; flores aperti, corollae laciniae late ovatae, breviter acutatae, $2,25 \mathrm{~mm}$ longae; ovarium 3 -ovulatum.

P. macrostachys Decne. nach Spegazzini in Nov. Add. Fl. Patag. II. (1902) 77, nicht $P$. macrostachys Decne. in Prodr.

Patagonien: Golfo de S. Jorge (Spegazzini 1899).

Die neue Art ist aus der Verwandtschaft von Plantago Candollei Rap.

Plantago Rojasii Pilger n. sp.; annua, radix fusiformis tenuis elongatus; folia numerosiora rosulata, tenuia, anguste obovato-ovalia, superne breviter late arcuato-cuneatim angustata, inferne sensim in petiolum brevem latum angustata, $10-12 \mathrm{~cm}$ longa, $3-3,5 \mathrm{~cm}$ lata, acute denticulata, supra adulta pilis longioribus villosis inspersa, ad marginem fere ciliata, infra imprimis ad nervos densius inspersa; pedunculi arcuatim adscendentes, ad $17 \mathrm{~cm}$ longi (cum spica ad 10 bis $11 \mathrm{~cm}$ longa), longius villosuli; spica angusta inferne parum tantum laxa; bractea anguste triangularis, pilis longis patulis inspersa, 2,25 ad $2,5 \mathrm{~mm}$ longa; sepala latiora late ovata, vix pilis inspersa, $2 \mathrm{~mm}$ longa, sepala angustiora anguste ovalia, pilis paucis brevibus inspersa; flores clausi, staminodia parum evoluta, laciniae corollae anguste ovatae vel lanceolato-ovatae, 2,25-2,5 mm longae; semina in capsula 3 .

Paraguay: am unteren Pilcomayo (Th. Rojas n. 219. - Juli 1906).

Die neue Art ist mit $P$. myosuros Lam. verwandt.

Plantago Stuckertii Pilger n. spec.; perennis, rhizomate brevi crassiusculo; folia ovalia, superne brevius arcuato-cuneatim angustata, inferne sensim in petiolum longiorem angustata, glabra, $19-20 \mathrm{~cm}$ longa, $3,5-4 \mathrm{~cm}$ lata, margine dentibus paucis magnis obtusis, $2-6 \mathrm{~mm}$ longis instructa; pedunculi erecti, $35 \mathrm{~cm}$ alti (cum spica $11 \mathrm{~cm}$ longa), inferne glabrescentes, superne albido-villosi; spica inferne laxa, superne densiuscula; bractea ovata, obtusa, margine brevissime vix lacerulatociliolulata, $2 \mathrm{~mm}$ longa; sepala latiora rotundata, satis inaequilatera, margine brevissime ciliolulata, dorso pilis nonnullis brevissimis obsita, 2,25 mm longa, sepala angustiora ovali-elliptica; flores aperti, corollae laciniae late ovatae, $2 \mathrm{~mm}$ longae; ovarium 3-ovulatum. 
Argentinien: Corảoba, Dep. Anejos Norte (Stuckert n. 3781. - November 1897); bei Cordoba (Stuckert n. 4963. - November 1898).

subsp. catamarcensis Pilger.; folia minora, 7-18 cm longa, cuneatim in petiolum brevem planum angustata; dentes in margine folii variantes, parce evolutae vel ad 2-2,5 mm longae; pedunculi ad $40 \mathrm{~cm}$ alti; bractea triangulari-ovata et sepala latiora late ovata quam in typo paulo angustiora.

Argentinien: Catamarca, Fuerte de Andalgalá (Schickendantz n. 228. - Februar 1876); Catamara, Quebrada de la Tala (Hieronymus et Lorentz s. n. - Februar 1872). 